\title{
Apoyo social percibido y dependencia funcional en el adulto mayor con dolor*
}

\author{
Perceived social support in the elderly with pain
}

\author{
Ara Mercedes Cerquera** \\ ORCID 0000 -0002 -6773-1495 \\ Ana Fernanda Uribe Rodríguez \\ ORCID 0000-0002-7275-5336 \\ Yeferson Jhair Matajira Camacho \\ ORCID $0000-0002-3900-1583$ \\ María Paula Delgado Porras \\ ORCID 0000-0001-9217-7089 \\ Universidad Pontificia Bolivariana \\ Recibido: 26 de noviembre de 2016 \\ Revisado: 20 de enero de 2017 \\ Aceptado: 3 de marzo de 2017
}

\section{Resumen}

En la actualidad la prevalencia de dolor en el adulto mayor constituye un problema de salud pública. Esta condición del doliente, en ocasiones tiende agravarse con el tiempo, limitan las actividades diarias del individuo y generan un impacto psicológico, social, familiar y laboral. El objetivo de este artículo es describir el nivel dependencia funcional y apoyo social percibido en el adulto mayor con dolor. Metodológicamente se concibe como una investigación transversal descriptiva con muestreo no probabilístico por conveniencia con una muestra de 200 adultos mayores de Bucaramanga. Se aplicó el instrumento de apoyo social percibido, la prueba Barthel y el cuestionario Mcguill. Como resultado se obtuvo que hay una mayor prevalencia de mujeres, casadas, en edades entre los 65 y 75 años, pertenecientes a estratos 3, con nivel de escolaridad sin finalización de básica primaria. Los participantes describen su dolor en mayor medida como brusco $58,5 \%$, cruel $71,5 \%$ y molesto $61,0 \%$. La mayoría tiene altos niveles de apoyo social y el $86,5 \%$ son totalmente independientes.

Palabras clave: adulto mayor, dolor, dependencia funcional, apoyo social.

Artículo de investigación. Este trabajo es producto del proyecto de la Dirección de Investigaciones de la Universidad Pontificia Bolivariana, código 096-0315-3100. DOI: http://dx.doi.org/10.15332/s1794-9998.2017.0002.06

** Correspondencia: Ara Mercedes Cerquera, Escuela de Ciencias Sociales, Universidad Pontificia Bolivariana, Bucaramanga, Santander, Colombia, dirección postal: Universidad Pontificia Bolivariana, Km. 7 Autopista a Piedecuesta, Oficina H-210, Floridablanca, Santander, Colombia Correo electrónico: ara.cerquera@upb.edu.co. 


\section{Abstract}

Currently, the prevalence of pain in the elderly constitutes a public health problem, since these disorders tend to worse over time, limiting the daily activities of the individual and generating a psychological, social, family and work impact. Objective: Describing the functional dependence level and the perceived social support in the elderly with pain. Methodology: transversal descriptive research with non-probability convenience sampling, with a sample of 200 older adults from bucaramanga. the instrument of perceived social support, the Barthel test and the McGill questionnaire were applied. The results indicate higher prevalence of $69 \%$ women, married, with ages between 65 and 75 years, belonging to social strata 3 , with a level of education without the elementary school complete. that participants describe their pain as abrupt 58.5\%, 71.5\% cruelly, 61.0\% Most have high levels of social and $86.5 \%$ is totally independent.

Keywords: Senior Adult, Pain, Functional Unit, Social support

\section{Introducción}

La idea de que la vejez es sinónimo de enfermedad ha prevalecido históricamente en la mente de los profesionales de la salud y población en general. Aunque la gerontología destaca lo fisiológico como una de las características principales del envejecimiento, también es una etapa de la vida donde crece la vulnerabilidad a padecer enfermedades y discapacidades (Ortigosa, Riquelme y Martín, 2010), por el declive físico, psicológico y social que trae consigo el aumento de la edad (Melguizo et al. 2014).

Con respecto a las principales afecciones crónicas que padece el adulto mayor, Durán, Valderrama, Uribe, González y Molina (2009) mencionan que son más comunes enfermedades cerebrovasculares, las demencias, y las enfermedades osteomuscular, mientras que para Barragán, Mejía y Gutiérrez (2007) son las cardiopatías, las enfermedades articulares, el cáncer y enfermedades óseas.

Dichas enfermedades se caracterizan por la presencia de dolor (Rodríguez, Esteve \& López 2000) que en el adulto mayor es de gran prevalencia el de tipo crónico (Acuña y Olivares, 2014; Durán, Orbegoz Valderrama, Uribe Rodríguez y Uribe Linde, (2008); Franco y Seone 2001; Morales y Cote, 2010; Thomas, Peat, Harris, Wilkie \& Croft, 2004), el cual hace referencia al dolor que persiste por más de un mes después de la lesión, y es considerado como un conjunto de manifestaciones biopsicosociales que carecen en ocasiones de propiedades fisiológicas reparadoras (López, Sánchez, Rodríguez y Vásquez, 2007). Sus consecuencias suelen estar asociadas a la manifestación de sufrimiento, problemas para la realización de actividades cotidianas, tanto del doliente (Barrios, Castilla, Montero y Rodríguez, 2011) como de su núcleo primario de interacción (Silvemark, Källmén, Portala, \& Molander, 2008).

Se ha intentado encontrar evidencia experimental que confirme la existencia de hipoalgesia en la edad avanzada, pero no ha sido confirmada a pesar de haberse encontrado umbrales de detección de dolor más altos en personas mayores utilizando estimulación térmica (Harkins, 1996 citado por López y Velasco, 2007). Por otra parte, existe evidencia de una actitud más conservadora en la persona mayor a la hora de etiquetar como doloroso un estímulo (López y Velasco, 2007).

En cuanto a los estudios epidemiológicos, mientras algunos señalan un aumento en la frecuencia del dolor según avanza la edad, otros indican un descenso en la frecuencia del dolor asociado a la edad, su prevalencia oscila entre el $22 \%$ y el $88 \%$. Parece que la prevalencia del dolor crónico en las personas mayores depende del tipo de dolor investigado y su localización (Casals \& Samper, 2004). 
Diferentes trabajos reportan menores frecuencias de dolor en personas mayores con respecto a cefaleas, dolor en pecho y estómago, y dolor muscular, pero sí mayores frecuencias de dolor de tipo articular en esta población (Bijlsma, 2002). Por otro lado algunas investigaciones señalan que existen diferencias fisiológicas, anatómicas, neurales, hormonales, psicológicas y socioculturales, que al ser tomadas en cuenta concluyen en los procesos de evaluación que la mujer reporta con mayor asiduidad dolor y presentan un umbral más bajo respecto a los hombres (Carneiro de Araújo, Ashmawi y Posso, 2011; Gutiérrez y Gutiérrez, 2012). De lo anterior cabe resaltar que uno de los avances con mayor relevancia en el estudio y tratamiento del dolor, ha sido el entendimiento adquirido, que respecta a la forma de evaluarlo y medirlo, válido tanto para los ensayos clínicos de técnicas analgésicas, como para la práctica clínica (Teniza, 2011).

De acuerdo al modelo biopsicosocial planteado por Gatchel, Peng, Peters, Fuchs y Turk (2007), el dolor es una respuesta dada por componentes neurofisiológicos, conductuales (motores) y subjetivos (verbales-afectivos) (Ruvalcaba y Domínguez, 2009) en la cual puede o no presentarse componentes patológicos pero que siempre tiene antecedentes y consecuentes fisiológicos (Flor \& Hermann, 2004). Por lo tanto, la atención del dolor crónico implica necesariamente la intervención farmacológica, médica y psicológica. Con respecto a este último, diversos autores como Gatchel y Okifuji, (2006); Sullivan, Feuerstein, Gatchel, Linton y Pransky (2005); Turk, (2004); Turner y Aaron, (2001); Turner, Jensen y Romano, (2000) hacen énfasis en el trabajo sobre la identificación y cambio de esquemas, pensamientos, creencias y emociones mal adaptativas, que el paciente tiene respecto a su condición por unos más positivos.

Como se puede identificar, existen diversos factores involucrados en la presencia de dolor (Londoño, Contreras, Delgadillo, Tobón y Vinaccia, 2005) y quizás uno de los más importantes es el entorno, y las relaciones interpersonales que el sujeto evalúa como significativas en su vida (Barra, 2004; Escobar, Puga y Martín, 2008) ya que para Santos (2009), las redes sociales ejercen influencia positiva en la salud y calidad de vida en los adultos mayores ya que contribuye a la supervivencia (Pinazo y Sánchez, 2005), en el sentido de que los demás, pueden ayudar para la satisfacción de necesidades básicas (Riquelme, Martín, Carrillo y López, 2010).

Aunque las investigaciones que tienen como evaluación el apoyo social y el dolor osteomuscular son escasas y no han sido claras en cuanto si una variable influye a la otra, se pudo encontrar en un estudio de 340 adultos mayores con dolor osteomuscular, alta percepción de apoyo social (Amaya y Carrillo, 2015). En cuanto a correlaciones entre dichas variables, Plata y Cerquera (2016) encontraron al evaluar 70 adultos mayores con dolor osteomuscular que no existía una correlación significativa entre variables y que se destacaba una percepción de apoyo social moderado. Adicional a lo anterior, es pertinente mencionar que pese a las escasas investigaciones, aún prevalecen diversos estereotipos en los que la vejez es vista como una etapa de desdicha, abandono, aislamiento, soledad, dolor y enfermedad (Carbajo, 2009; Aristizabal, Morales, Salas y Torres, 2009).

Ahora bien, esta presencia de dolor junto con los diversos cambios que trae consigo la vejez, genera en la mayoría de las ocasiones un deterioro en las capacidades funcionales (Araña-Suárez, 2011), lo que genera un estado de dependencia entendido como la imposibilidad del sujeto a valerse por sus propios recursos para realizar las tareas cotidianas (Manrique-Espinoza, Salinas-Rodríguez, Moreno y Téllez-Rojo, 2011; Bertone, Torres y Andrada, 2010; Jiménez et al., 2012; Barrios, Castilla, Montero y Rodríguez, 2011).

No obstante, cabe resaltar que para algunos autores como Manrique et al., (2011) la edad no está relacionada con la dependencia, pues el estado de salud en los adultos mayores, es el resultado de múltiples variables como los hábitos alimenticios, el ejercicio físico, las actividades de ocio, etc. Sin embargo, en estudios como el de Gázquez, Pérez, Mercader y Molero (2011), se evidencia una correlación positiva, entre el aumento de la edad y el aumento de la dependencia, argumento compartido por Segovia y Torres (2011); González y Ham-Chande 
(2007) y por los estudios realizados por Pinillos y Prieto (2012), que expresan una relación existente entre la dependencia tanto en las actividades básicas de la vida y las instrumentales, que hacen alusión a medios que posibilitan la relación con el entorno (Ávila, Melano, Payette y Amieva, 2007); (Alves y Rodríguez, 2005).

Por otro lado, Moscoso (2013); Díaz y Marulanda (2011); Noceda, Moret y Lauzirika (2006); Soto y Barrios (2012) mencionan que la situaciones de dependencia en el adulto mayor son producto de afecciones de salud, lo cual concuerda con las investigaciones realizadas por Abellán, Esparza y Pérez (2011), quienes al trabajar con una población española encontraron que más del $48,7 \%$ de los adultos mayores presentaba algún tipo de dolencia y también con los postulados de Alves y Rodríguez (2005) que mencionan que existe una relación causal entre el proceso de envejecimiento, el dolor y la presencia de enfermedades crónicas, y la dependencia funcional.

Sin embargo cabe resaltar, que existe una dificultad para establecer una relación directa entre la enfermedad y el deterioro funcional, pues en muchas ocasiones de una patología leve, puede darse un deterioro mayor a nivel funcional, que de una enfermedad de peor pronóstico (Barrantes, García, Gutiérrez y Miguel 2007). Por ello, resulta de gran utilidad realizar una adecuada evaluación de la capacidad funcional, con el fin de orientar intervenciones y su respectivo acompañamiento (Alonso, Tirado, Van-der y Rodríguez, 2014).

Es por lo anterior que el objetivo de la presente investigación es describir el nivel de dependencia funcional y apoyo social percibido en el adulto mayor con dolor osteomuscular de la ciudad de Bucaramanga.

\section{Método}

Enfoque cuantitativo, de alcance descriptivo, con un diseño no experimental de corte transversal (Hernández, Fernández y Baptista, 2014).

\section{Participantes}

Se trabajó con una muestra de 200 adultos mayores de Bucaramanga que referían padecer dolor. Se utilizó un muestreo no probabilístico a conveniencia usando el método de recolección voz a voz. Los criterios de inclusión fueron: (a) edad mayor o igual a 65 años, (b) tener capacidad y voluntad de responder los cuestionarios, (c) que auto reporten padecer de dolor osteomuscular, (d) que refieran haber padecido dolor en el mes en que se realiza el estudio, (e) tener capacidad de firmar el consentimiento informado. Se excluyeron del estudio aquellos adultos mayores que: (a) presentaran una incapacidad física o mental que les dificultara responder los cuestionarios.

\section{Procedimiento}

Inicialmente se creó una estrategia de contacto voz a voz con los estudiantes de la Universidad Pontificia Bolivariana, con los cuales se entró en contacto con sus familiares adultos mayores, posteriormente se contactó con la muestra vía telefónica y se concretaron fechas de encuentro para la aplicación de los instrumentos psicométricos. En la investigación se aseguró la confidencialidad de la participación de todas las personas, mediante un consentimiento informado, en el que se indicaba que la información obtenida era de uso exclusivamente académico y sus nombres mantenidos en el anonimato. Lo anterior bajo la Ley 1090 de 2006, numeral 9, del Artículo 2, que hace referencia a la investigación con humanos y el respeto a la dignidad y el bienestar de las personas que participan con conocimiento en una investigación. Del mismo modo, se tuvo en cuenta el Artículo 50 que dicta que los profesionales de la psicología, al llevar a cabo investigaciones científicas, deberán basarse en principios éticos de respeto y dignidad, lo mismo que salvaguardar el bienestar y los derechos de los participantes. Posterior a la aplicación de las pruebas, se dio lugar al análisis de los resultados mediante el programa estadístico spss 22 en el cual se tabularon los datos y se hicieron los diferentes análisis descriptivos de las variables de trabajo. 


\section{Instrumentos}

\section{Cuestionario de Dolor de McGill (Bejarano, Osorio, Rodríguez y Berrio 1985)}

Instrumento que consta de 61 adjetivos distribuidos en 20 grupos, incluyendo cada grupo de 2 a 4 adjetivos que califican la experiencia dolorosa. Cada uno de los términos descriptivos tiene asignado un número o rango que permite obtener una puntuación de acuerdo a las palabras escogidas, con lo que se obtiene el denominado "Pain Rating Index" (PRI) o “Índice de Valoración del Dolor”. Esta puntuación refleja el modo en que el paciente califica su propia experiencia dolorosa, teniendo en cuenta factores emocionales y sensoriales. Además, contiene un apartado en cual el paciente refleja la intensidad del dolor que padece, el "Present Pain Intensity " (PPI) o "Índice de Intensidad del Dolor" (Ítem 21); una figura humana para marcar las zonas donde siente dolor (ítem 22), y los síntomas que acompañan el dolor (ítem 23). Las propiedades psicométricas del MPQ varían en diferentes grupos de pacientes (Bernt, 1991, citado en Boyle, Fernández y Ortet, 2003). Al ser una prueba clínica la confiablidad de este instrumento en interna se mide por la comprensión de los descriptores y los valores encontrados por ítem, tal y como lo señalan los autores del instrumento.

\section{Cuestionario mos de apoyo social percibido. (Londoño et al., 2012)}

Validado en Colombia, es un instrumento que contiene 20 preguntas, con opción de respuesta tipo Likert: nunca (1), pocas veces (2), algunas veces (3), la mayoría de las veces (4), siempre (5), de manera que a mayor puntuación, mayor apoyo social percibido. Los ítems evalúan cuatro tipos de apoyo: apoyo emocional o informacional, que se refiere a la comprensión empática y la guía u oferta de consejos y orientación (preguntas 3, 4, 8, 9, 13, 16, 17, 19, punto de corte: 24 puntos); apoyo instrumental, es decir, la provisión de ayuda material que pueda precisar la persona (preguntas: 2, 5, 12, 15, punto de corte:12 puntos); interacción social positiva, referida a la disponibilidad de personas con las cuales salir, divertirse o distraerse (preguntas 7, 11, 14,18 , punto de corte:12 puntos); y apoyo afectivo, la expresión de amor y afecto (preguntas 6, 10, 20, punto de corte: 9 puntos).

\section{Índice de Barthel (Shah, Vanclay \&Cooper, 1989)}

Diseñado en 1955 y publicado en 1965 por Mahoney y Barthel. Para esta investigación se utilizó la última versión actualizada del instrumento realizada por Shah S. Vanclay F, Cooper B en 1989. Su propósito es evaluar el estado funcional de cada paciente ante el riesgo de discapacidad, de modo que mide la capacidad de una persona para realizar diez actividades de la vida diaria (AVD), consideradas como básicas, lo que permite obtener una estimación cuantitativa de su grado de independencia, para conocer la funcionalidad del paciente en relación a las actividades de la vida diaria. Cada actividad de la vida diaria comprende 5 rangos de respuesta, donde 1 representa totalmente independencia, 2 necesita ayuda mínima, 3 moderada ayuda. 4 gran ayuda, 5 dependiente. Confiabilidad del instrumento 0,92 .

\section{Resultados}

En la tabla 1, el $69 \%$ corresponde a las mujeres y el $30 \%$ a los hombres, con edades comprendidas en su mayoría $58,5 \%$ entre los 65 y 75, $41,0 \%$ son casados, $65,0 \%$ pertenecientes a estrato 3 , con un vínculo laboral asociado a la realización de tareas propias del hogar $36 \%$ y con un nivel de escolaridad sin finalización de la básica primaria $43,0 \%$. El $72 \%$ de la población desconoce su diagnóstico médico respecto a sus dolencias.

Respecto a la tabla 2 se observa que la muestra describe su dolor como brusco $58,5 \%$, cruel $71,5 \%$, molesto $61,0 \%$, de intensidad fuerte $38,5 \%$, con afectación disminuida de la actividad diaria en el $58,0 \%$ y con un ciclo del sueño normal en un $63,0 \%$ 
Tabla 1.

Descripción sociodemográfica del total de la muestra

\begin{tabular}{|c|c|c|c|}
\hline & Descripción & $\mathrm{Fi}$ & $\%$ \\
\hline \multirow{2}{*}{ Sexo } & Masculino & 61 & 30,5 \\
\hline & Femenino & 139 & 69 \\
\hline \multirow{3}{*}{ Edad } & 65 a 75 años & 117 & 58,5 \\
\hline & 76 a 84 años & 64 & 32,0 \\
\hline & 85 años hacia adelante & 19 & 9,5 \\
\hline \multirow{6}{*}{ Estado civil } & Casado & 82 & 41,0 \\
\hline & Soltero & 38 & 19,0 \\
\hline & Viudo & 60 & 30,0 \\
\hline & Divorciado & 5 & 2,5 \\
\hline & Unión no formalizada & 5 & 2,5 \\
\hline & Separado & 10 & 5,0 \\
\hline \multirow{3}{*}{ Estrato social } & 2 & 40 & 25,0 \\
\hline & 3 & 150 & 65,0 \\
\hline & 4 & 10 & 10,0 \\
\hline \multirow{6}{*}{ Vínculo laboral } & V. laboral estable & 7 & 3,5 \\
\hline & V. laborar inestable & 12 & 6,0 \\
\hline & Pensionado jubilado & 60 & 30,0 \\
\hline & Actividad no lucrativa & 2 & 1,0 \\
\hline & Tareas propias del hogar & 72 & 36,0 \\
\hline & Otro & 46 & 23,0 \\
\hline \multirow{8}{*}{ Escolaridad } & No sabe leer ni escribir & 9 & 4,5 \\
\hline & No terminó la básica primaria & 86 & 43,0 \\
\hline & Básica primaria terminada & 51 & 25,5 \\
\hline & No terminó la enseñanza media & 24 & 12,0 \\
\hline & Terminó la enseñanza media & 13 & 6,5 \\
\hline & Enseñanza media superior & 11 & 5,5 \\
\hline & Universitario & 3 & 1,5 \\
\hline & Desconoce & 3 & 1,5 \\
\hline \multirow{9}{*}{ Diagnóstico } & Artrosis & 21 & 10,5 \\
\hline & Osteoporosis & 23 & 11,5 \\
\hline & Fibromialgia & 3 & 1,5 \\
\hline & Osteo artrosis & 1 & 0,5 \\
\hline & Artritis & 4 & 2,0 \\
\hline & Desconoce & 145 & 72,0 \\
\hline & Línea discal & 1 & 0,5 \\
\hline & Contracción muscular & 1 & 0,5 \\
\hline & $\begin{array}{l}\text { Osteo artritis } \\
\text { Descalcificación }\end{array}$ & 1 & 0,5 \\
\hline
\end{tabular}

Número de participantes de la investigación: 200 adultos mayores

Edad media: 74.02 
Tabla 2.

Valoración cuantitativa del dolor desde las cuatro dimensiones - McGill-Melzack

\begin{tabular}{|c|c|c|c|}
\hline Dimensión & & $\mathrm{Fi}$ & $\%$ \\
\hline \multirow{12}{*}{ Sensorial } & Que pulsa & 81 & 69,0 \\
\hline & Brusco & 117 & 58,5 \\
\hline & Que perfora & 86 & 43,0 \\
\hline & Punzante & 91 & 45,5 \\
\hline & Que presiona & 108 & 54,0 \\
\hline & Que estira & 86 & 43,0 \\
\hline & Ardor & 46 & 23,0 \\
\hline & Hormigueo & 46 & 23,0 \\
\hline & Resentido & 83 & 41,5 \\
\hline & Tenso & 84 & 42,0 \\
\hline & Fatigante & 30 & 15,0 \\
\hline & Enfermante & 44 & 22,0 \\
\hline \multirow{3}{*}{ Afectiva } & Deprimente & 50 & 25,0 \\
\hline & Cruel & 143 & 71,5 \\
\hline & Desesperante & 98 & 49,0 \\
\hline \multirow{2}{*}{ Evolutiva } & Molesto & 122 & 61,0 \\
\hline & Que penetra & 127 & 63,5 \\
\hline \multirow{3}{*}{ Miscelánea } & Rígido & 39 & 19,5 \\
\hline & Frio & 24 & 12,0 \\
\hline & Leve & 19 & 9,5 \\
\hline \multirow{6}{*}{ Intensidad del dolor actualmente } & Moderado & 73 & 36,5 \\
\hline & Fuerte & 77 & 38,5 \\
\hline & Intenso & 15 & 7,5 \\
\hline & Insoportable & 16 & 8,0 \\
\hline & Normal & 73 & 36,5 \\
\hline & Disminuida & 116 & 58,0 \\
\hline \multirow[t]{2}{*}{ Actividad diaria } & Mínima & 11 & 5,5 \\
\hline & Normal & 126 & 63,0 \\
\hline \multirow[t]{2}{*}{ Sueño } & Interrumpido & 62 & 31,0 \\
\hline & Sueño & 12 & 6,0 \\
\hline
\end{tabular}


Tabla 3.

Prueba índice de Barthel. Nivel de dependencia funcional

\begin{tabular}{lcr}
\hline & Fi & $\%$ \\
\hline 61 a 90 dependencia moderada & 12 & 6,0 \\
\hline 91 a 99 dependencia escasa & 15 & 7,5 \\
\hline Totalmente independiente & 173 & 86,5 \\
\hline
\end{tabular}

Con respecto a la variable dependencia funcional se puede evidenciar que el $86,5 \%$ de la población es totalmente independiente, frente a un bajo porcentaje de 6,0\% que presenta dependencia moderada y $7,5 \%$ que manifiesta dependencia escasa.

Tabla 4.

Valoración cuantitativa de los tipos de apoyo social percibido MOS

\begin{tabular}{llcc}
\hline \multicolumn{1}{c}{ Tipos de apoyo } & & Fi & $\%$ \\
\hline $\begin{array}{l}\text { Emocional/ } \\
\text { Informacional }\end{array}$ & Adecuado uso & 162 & 72,0 \\
\cline { 2 - 4 } & Escaso uso & 38 & 28,0 \\
\hline \multirow{2}{*}{ Instrumental } & Adecuado uso & 167 & 76,0 \\
\cline { 2 - 4 } & Escaso uso & 33 & 24,0 \\
\hline Interacción & Adecuado uso & 164 & 73,0 \\
\cline { 2 - 4 } social positiva & Escaso uso & 36 & 27,0 \\
\hline \multirow{2}{*}{ Afectivo } & Adecuado uso & 169 & 78,0 \\
\cline { 2 - 4 } & Interrumpido & 31 & 22,0 \\
\hline
\end{tabular}

En relación con el cuestionario mos de apoyo social percibido, se puede observar en la tabla 4 que la muestra tiene una buena percepción del apoyo afectivo 78,0 \%, seguido del apoyo instrumental $76,0 \%$, interacción positiva $73,0 \%$ y apoyo informacional $72,0 \%$.

\section{Discusión}

El dolor osteomuscular en la población geriátrica, ha llevado a que este sea considerado como problema sanitario a nivel mundial por su proporción epidémica, debido a la afectación que trae consigo en la calidad de vida del individuo y disminución de su funcionalidad, así como, a nivel económico, producto del costo de la atención médico-asistencial; por ello, la mayor parte de las investigaciones se han enfocado en describir el fenómeno en función a sus características y establecimiento de parámetros para su diagnóstico y tratamiento. Sin embargo, pocos estudios han abordado el dolor osteomuscular, al considerar aspectos psicosociales propios de su esfera individual, motivo por el cual se planteó como objetivo de investigación la descripción del nivel de dependencia funcional y apoyo social percibido en el adulto mayor con este tipo de dolor que atañe a la población.

En relación con las características sociodemográficas de la muestra estudiada, se encontró que estaba constituida en mayor proporción por mujeres (69\%) que hombres (30,5\%), con edades comprendidas entre los 65 y 75 años de edad, los cuales presentaban un nivel de educación bajo, donde el $43 \%$ no terminó la básica primaria, y actualmente se encargan de las tareas del hogar; tales resultados concuerdan con los datos constatados en el estudio de Plata y Cerquera (2016), donde la muestra estaba constituida de igual manera en mayor proporción por mujeres $(86,1 \%)$, quienes presentan una básica primaria $(44,4 \%)$, teniendo como vínculo laboral tareas del hogar en un $41,6 \%$, desempeñándo tareas como ser amas de casa (69\%); lo anterior, parecería confirmar la relación que Lobera y García en el 2014 señalaron, al considerar que las personas que llevan a cabo actividades domésticas que impliquen una reiteración. Al ser por lo general mujeres a quienes se les adjudica el desempeño de tareas domésticas, son las que reportan con mayor frecuencia dolor osteomuscular; relación también identificada por Robaina y Sevilla en el 2003. De igual manera, la percepción del mismo dolor y su nivel educativo parecería mantener una relación en cuanto al manejo y evaluación de su dolor (Asociación Colombiana para el Estudio del Dolor (ACED), 2008 y Manson, 2010).

En cuanto a la variable de apoyo social percibido, el cual hace alusión a la seguridad por parte del individuo ante la disponibilidad de apoyo una vez lo solicite (Londoño et al., 2012), se identificó que los participantes obtuvieron elevados porcentajes en función del uso adecuado de los diferentes tipos de apoyo (afectivo, $78 \%$, instrumental $76 \%$, interacción positiva $73 \%$ e informacional $72 \%$ ), lo cual es acorde con lo señalado por Pinazo y Sánchez (2005), al considerar que las relaciones significativas en la vida 
del individuo inciden principalmente en su calidad de vida y su condición actual de salud, al provisionar herramientas que satisfagan sus necesidades, posibilitando los procesos de adaptación personal, familiar y social, lo cual se evidenció de igual manera en los estudios desarrollados por Riquelme, Martín, Carrillo y López en el (2010) y Rodrigo y Byrne (2011). Sin embargo, cabe resaltar que en relación al apoyo social y el dolor osteomuscular son limitadas las investigaciones que hayan hecho estudios en donde correlacionen estas dos variables, lo cual ha imposibilitado establecer si prevalece una influencia determinante, puesto que, los resultados obtenidos en estos estudios, se ha evidenciado tanto correlaciones significativas (Amaya y Carrillo, 2015), como no significativas (Plata y Cerquera, 2016).

Respecto a la valoración cuantitativa del dolor, se evidenció que la mayor proporción de la población describe como cruel su dolor con un $71,5 \%$, en la dimensión afectiva, la cual reseña este en términos de estrés, asociado a emociones y temores, seguido de un 61 \% en la dimensión evolutiva, descrito como molesto, considerando el dolor desde aspectos subjetivos de su experiencia. Los anteriores resultados obtenidos podrían estar relacionados con el desconocimiento ante su diagnóstico, en donde el $72 \%$ de la población lo indicó. Autores como Blazer (2000) y Cornachione (2006), justifican lo anterior al señalar que el adulto mayor, en esta etapa de la vida, está sujeto a presentar niveles de estrés elevados junto al aumento de presión arterial, por el conjunto de cambios que debe enfrentar a nivel fisiológico, laboral y psicosocial, los cuales son incontrolables e irreversibles, conllevando a la dependencia y trastornos del estado de ánimo.

Sin embargo, autores como Hernández y Romero (2010) señalan que es necesario tener en cuenta aspectos tales como actividades de ocio, hábitos alimenticios, ejercicio y la percepción subjetiva del individuo, lo cual, influirá en la consecución de recursos para manejar el estrés; así como su capacidad para desarrollar actividades de la vida diaria, considerando este como su estado funcional, las cuales no están directamente relacionadas con la edad (Manrique et al., 2011); lo expresado anteriormente, se consolida en esta investigación puesto que el $86,5 \%$ de la población fue totalmente independiente a diferencia de un $6 \%$,que representó una dependencia escasa.

Se recomienda realizar posteriormente un estudio comparativo en función al género, el cual tenga como objetivo, identificar si existe una diferencia en cuanto a las características del dolor (intensidad, percepción a nivel emocional y sensorial, síntomas) y el estado funcional del individuo, implementando las mismos instrumentos de evaluación de esta investigación, que han sido sugeridos por el Consenso Interdisciplinario para la evaluación del dolor en el adulto mayor, ya que en precedentes estudios se hallaron diferencias fisioanatómicas, neuronales, hormonales y psicosociales (Carneiro de Araújo, Ashmawi \& Posso, 2011; Gutiérrez y Gutiérrez, 2012).

Finalmente, es relevante señalar la necesidad de continuar desarrollando Estudios descriptivos que posibiliten ahondar en aspectos psicosociales de la población del adulto mayor y su implicación a nivel individual, con el propósito de caracterizar estas variables y en estudios correlaciónales posteriores dar a conocer si existe relación alguna.

\section{Referencias}

Abellán, A., Esparza, C., y Pérez, J. (2011). Evolución y estructura de la población en situación de dependencia. Cuadernos de relaciones laborales, 29(1), 93-123.

Acuña, J. y Olivares, A. (2014). Dolor en el paciente de la tercera edad. Revista Médica Clínica las Condes, 25(4), 674-686.

Alonso, M., Tirado, S., Van-der, C., y Rodríguez, J. (2014). Evaluación de un programa de intervención grupal en dolor crónico y fibromialgia. Revista Psicología de la Salud, 2(1), 42-75.

Alves, L., y Rodríguez, R. (2005). Autopercepción de determinante de la salud entre las personas mayores en Sao Paulo. Salud Pública, 17(5), 333-341.

Amaya, M., y Carrillo, G. (2015). Apoyo social percibido y afrontamiento en personas con dolor crónico no maligno. Aquichan, 15(4), 461-474. 
Araña-Suárez, S. (2011). Trastornos musculo-esqueléticos, psicopatología y dolor. Recuperado de http://www.seg-social.es/prdi00/groups/ public/documents/binario/143942.pdf

Aristizábal, N., Morales, A., Salas, B. y Torres, A. (2009). Estereotipos negativos hacia los adultos mayores en estudiantes universitarios. Cuadernos Hispanoamericanos de Psicología, 9(1), 35-44.

Asociación Colombiana para el Estudio del Dolor (ACED) (2008). Estudio Nacional del Dolor. Recuperado de http://www.dolor.org.co/ index.php?option=com_content\&view=article \&id $=68 \&$ ttemid $=88$.

Ávila, F., Melano, C., Payette, H., y Amieva, H. (2007). Síntomas depresivos como factor de riesgo de dependencia en adultos mayores. Salud pública, 49(5), 367-375.

Barra, E. (2004). Apoyo social, estrés y salud. Psicología y Salud, 14(2), 237-243.

Barragán, A., Mejía, S., y Gutiérrez, L. (2007). Dolor en adultos mayores de 50 años: prevalencia y factores asociados. Salud Pública de México, 48, 488-494.

Barrantes-Monge, M., García-Mayo, E.J., GutiérrezRobledo, L.M., y Miguel-Jaimes, A. (2007). Dependencia funcional y enfermedades crónicas en ancianos mexicanos. Salud Pública de México, 49, 459-466.

Barrios, Z., Castilla, J., Montero, K., y Rodríguez, R. (2011). Manejo integral del adulto mayor con dolor crónico, en el asilo san Pedro Claver basado en la teoría de Virginia Henderson. Revista Ciencia y Salud Virtual, 3(1), 113-122.

Bejarano, P.F., Osorio, R., Rodríguez, M.L., y Berrío, G. M. (1985). Evaluación de dolor: adaptación del cuestionario de McGill. Revista Colombiana de Anestesiología, 13(4), 321-51.

Bertone, C.L., Torres, V.E., y Andrada, M. (2014). Factores sociales e individuales que explican la dependencia funcional de los adultos mayores de cuatro ciudades latinoamericanas. Revista Científica de la Universidad Nacional de La Rioja, 1(3), 13-20.

Bijlsma, J. (2002). Analgesia and the patient with osteoarthritis. American Journal Therapy, 9(3), 189-197.

Blazer, D. (2000). Psiquiatría geriátrica. En Tratado de psiquiatría (pp. 46-61). Barcelona: Ediciones Hales/ Udoksky/Masson.

Boyle, G.J., Fernández, E., y Ortet, G. (2003). El cuestionario de dolor de McGill (McGill Pain Questionnaire-MPQ): consideraciones lingüísticas y estadísticas The Mc Gill Pain QuestionnaireMPQ: Linguistic and Statistical Consideration. Revista de Psicología de la Universidad de Chile, 12(1), 111-119.

Carbajo, M. (2009). Mitos y estereotipos sobre la vejez. Propuesta de una concepción realista y tolerante. Ensayos, (24), 87-96.

Carneiro de Araujo, C., Ashmawi, H. y Posso, I. (2011). Sexo y percepción del dolor y analgesia. Revista Brasileira de Anestesiología, 61(6), 449-458.

Casals M., \& Samper D. (2004). Epidemiology, prevalence and quality of life of non-malignant chronic pain. ITACA study. Revista Sociedad Española del Dolor, 11, 260-269

Cornachione, M. (2006). Psicología del desarrollo. Vejez: aspectos biológicos, psicológicos y sociales. Córdoba: Brujas.

Díaz, R. y Marulanda, F. (2011). Dolor crónico nociceptivo y neuropático en población adulta de Manizales (Colombia). Acta Medica Colombiana, 36(1), 10-17.

Durán, A., Valderrama, L., Uribe, A., González, A., y Molina, J. (2009). Enfermedad crónica en adultos mayores. Universitas Médica, 51(1), 16-28.

Durán, D., Orbegoz, L., Uribe, A. y Uribe, J. (2008). Integración social y habilidades funcionales en adultos mayores. Universitas Psychologica, 7(1), 263-270. 
Escobar, M.A., Puga, D. y Martín, M. (2008). Asociaciones entre la red social y la discapacidad al comienzo de la vejez en las ciudades de Madrid y Barcelona en 2005. Revista Española de Salud Pública, 82(6), 637-651.

Flor, H. \& Hermann, C. (2004). Byopsicosocial models of pain. En R.H., Dworkin \& W.S. Breitbart (Eds.): Psychosocial aspects of pain: A handbook for health care providers. Progress in pain research and management, 27, 47-78.

Franco, M., y Seoane, A. (2001). Características del dolor crónico en el anciano: tratamiento. Revista de la Sociedad Española de Dolor, 8(1), 29-38.

Gatchel, R.J. \& Okifuji, A. (2006). Evidence-based scientific data documenting the treatment and cost-effectiveness of comprehensive pain programs for chronic pain management. The Journal of Pain, 7, 779-793.

Gatchel, R.J., Peng, Y.B., Peters, M.L., Fuchs, P.N. \& Turk, D.C. (2007). The biopsychosocial approach to chronic pain: Scientific advances and future directions. Psychological Bulletin, 13(4), 581-624.

Gázquez, J., Pérez, M., Mercader, I., y Molero, M. (2011). Prevalencia de la dependencia funcional en personas mayores. Anales de Psicología, 27(3), 871-876.

González, C., y Ham-Chande, R. (2007). Funcionalidad y salud: una tipología del envejecimiento en México. Salud Pública de México. 49(4), 448-458.

Gutiérrez, W., y Gutiérrez, S. (2012). Diferencias de sexo en el dolor. Una aproximación a la clínica. Revista Colombiana de Anestesiología, 40(3), 207-212.

Hernández, R., Fernández, C., y Baptista, P. (2014). Metodología de investigación. México: Editorial Mc Graw Hill.

Hernández, Z. y Romero, E. (2010). Estrés en personas mayores y estudiantes universitarios: un estudio comparativo. Psicología Iberoamericana, 18(1), 56-68.
Jiménez-Caballero, P., López-Espuela, F., PortillaCuenca, J., Pedrera-Zamorano, J., JiménezGracia, M., Lavado-García, J.M. y CasadoNaranjo, I. (2012). Valoración de las actividades instrumentales de la vida diaria tras un ictus mediante la escala de Lawton y Brody. Revista de Neurología, 55(6), 337-342.

Lobera, J. y García. (2014). Identidad, significado y medición de las amas de casa. Cuadernos de Psicología, 16(1), 213-226.

Londoño, C., Contreras, F., Delgadillo, G., Tobón, S. y Vinaccia, S. (2005). Barreras para el manejo efectivo del dolor crónico. Revista de Psicopatología y Psicología Clínica, 10(1), 25-31.

Londoño, N., Rogers, H., Castilla, F., Posada, S., Ochoa, N., Jaramillo, M., Aguirre, D. (2012). Validación en Colombia del cuestionario MOS de apoyo social. International Journal of Psychological Research, 5(1), 142-150.

López, A., y Velasco, L. (2007). Dolor y envejecimiento. Informes Portal Mayores, 70.

López-Silva, M.C., Sánchez de Enciso, M., RodríguezFernández, M.C. y Vázquez-Seijas, E. (2007). Cavidol: calidad de vida y dolor en atención primaria. Revista de la sociedad Española del Dolor, 14(1), 9-19.

Manrique-Espinoza, B., Salinas-Rodríguez, A., Moreno-Tamayo, K., y Téllez-Rojo, M. (2011). Prevalencia de dependencia funcional y su asociación con caídas en una muestra de adultos mayores pobres en México. Salud Pública de México, 53(1), 26-33.

Manson, J.E. ( 2010). Pain: sex differences and implications for treatment. Metabolism: clinical and experimental. 59, 16-20.

Melguizo, E., Ayala, S., Grau, M., Merchán, A., Montes, T., Payares, C. y Reyes, T. (2014). Calidad de vida de adultos mayores en centros de protección social en Cartagena (Colombia). Aquichan, 14(4), 537-548.

Morales, J., y Cote, E. (2010). Características clínicas del dolor en adultos mayores de la 
Fundación Santa Sofía en Bogotá D.C. Revista UDCA Actualidad \& Divulgación Científica,13(2), 7-14.

Moscoso, J. (2013). El dolor crónico en la historia. Revista de Estudios Sociales, (47), 170-176.

Noceda, J., Moret, C., y Lauzirika, I. (2006). Características del dolor osteomuscular crónico en pacientes de atención primaria: resultados de un centro rural y otro urbano. Revista de la Sociedad Española del Dolor, 13(5), 287-293.

Ortigosa J., Riquelme M., Y Martín M. (2010) Manual de psicogerontología. Madrid: Editorial Académica S.A.

Pinazo, S., y Sánchez, M. (2005). Gerontología: actualización, innovación y propuestas. El apoyo social y las relaciones sociales de las personas mayores. Madrid: Pearson Educación.

Pinillos, Y. y Prieto, E. (2012). Funcionalidad física de personas mayores institucionalizadas y no institucionalizadas en Barranquilla, Colombia. Salud Pública. 14(3), 436-445.

Plata-Osma, L.J. y Cerquera-Córdoba, A.M. (2016). Dolor osteomuscular y apoyo social en una muestra de gerontes de Bucaramanga. Pensamiento Psicológico, 14(2), 125-135.

Riquelme, A., Martín, M., Carrillo, M. y López, A. (2010). Apoyo social en ancianos. En A. Riquelme., J. Ortigosa y M. Martín (Eds.), Manual de psicogerontología (pp.145-164). Murcia: Ediciones Académicas.

Rodrigo, M.J. \& Byrne, S. (2011). Social support and personal agency in at-risk mothers. Psychosocial Intervention, 20(1), 13-24.

Rodríguez, M., Esteve, R. \& López, A. (2000). Represión emocional y estrategias de afrontamiento en dolor crónico oncológico. Psicothema, 12(3), 339-345.

Robaina, C. y Sevilla, D. (2003). Epidemiología de las enfermedades relacionadas con la ocupación. Revista Cubana de Medicina General Integral,
19(4). Recuperado de http://www.bvs.sld.cu/ revistas/mgi/vol19_4_03/mgi10403.htm.

Ruvalcaba, G., y Domínguez, G. (2009). La terapia psicológica del dolor crónico. Psicología y Salud,19(2), 247-252.

Santos, Z. (2009). Adulto mayor, redes sociales e integración. Revista Trabajo Social, 2(11), 159-174.

Segovia, M. y Torres, E. (2011). Funcionalidad del adulto mayor y el cuidado enfermero. Gerokomos, 22(4), 162-166.

Shah, S., Vanclay, F. \& Cooper, B. (1989). Improving the sensitivity of the Barthel Index for stroke rehabilitation. Journal of Clinical Epidemiology, 42(8), 703-709.

Silvemark, A., Källmén, H., Portala, K. \& Molander, C. (2008). Life satisfaction in patients with longterm non-malignant pain-relating LiSat-11 to the Multidimensional Pain Inventory (MPI). Health \& Quality of Life Outcomes, 61-10.

Soto, O., y Barrios, S. (2012). Caracterización de salud, dependencia, inmovilidad y riesgo de úlceras por presión de enfermos ingresados al programa de atención domiciliaria. Ciencia y Enfermería, 18(3), 61-72.

Sullivan, M.J., Feuerstein, M., Gatchel, R., Linton, S.J., \& Pransky, G. (2005). Integrating psychosocial and behavioral interventions to achieve optimal rehabilitation outcomes. Journal of Occupational Rehabilitation, 15(4), 475-489.

Teniza, D. (2011). Valoración del dolor en el paciente adulto con afección cardiovascular. Revista Mexicana de Enfermería y Cardiología, 19(1), 34-40.

Thomas, E., Peat, G., Harris, L., Wilkie, R. \& Croft, P. (2004). The prevalence of pain and pain interference in a general population of older adults: cross-sectional findings from the North Staffordshire Osteoarthritis. Pain, 110(1), 361-368. 
Turk, D.C. (2004). Understanding pain sufferers: the role of cognitive processes. The Spine Journal, 4(1), 1-7.

Turner, J.A. \& Aaron, L.A. (2001). Pain-related catastrophizing: what is it? The Clinical Journal of Pain, 17(1), 65-71.
Turner, J.A., Jensen, M.P. \& Romano, J.M. (2000). Do beliefs, coping, and catastrophizing independently predict functioning in patients with chronic pain? Pain, 85(1), 115-125. 